\title{
Motivationale Faktoren im Wirkungskontext von Forschendem Lernen
}

\section{Carmen Wulf, Janina Thiem und Christopher Gess}

In der Diskussion um Forschendes Lernen wird davon ausgegangen, dass es einerseits ein besonders geeignetes Format zur Förderung unterschiedlicher (Forschungsund Schlüssel-)Kompetenzen darstellt, andererseits dieses Format aber in enger Verbindung zur Ausprägung der Lern- und Forschungsmotivation Studierender steht. Im Beitrag wird der Förderung von kognitiven und affektiv-motivationalen Forschungskompetenzen sowie der allgemeinen autonomen (Lern-)Motivation durch ein Format Forschendes Lernens in einem Prä-Post-Kontrollgruppendesign nachgegangen und Zusammenhänge zwischen Kompetenzveränderung und Motivation geprüft.

\section{$1 \quad$ Einleitung}

Neben der Förderung und Aneignung fachspezifischer Lerninhalte zielen Formate Forschenden Lernens insbesondere auf die Vermittlung von Forschungskompetenzen sowie einer forschenden Haltung ab (Huber, 2004; Gess, Deicke \& Wessels, 2017). Zusätzlich wird davon ausgegangen, dass sich mit dieser Lehr-Lern-Form die Motivation und die Selbststeuerung der Studierenden sowie diverse Schlüsselkompetenzen wie beispielsweise Problemlösungs-, Team- oder Kommunikationsfähigkeit fördern lassen (Wissenschaftsrat, 2006, 2015; Huber, 2009; Schubarth \& Speck, 2012). 
Mittlerweile lassen sich einige Studien mit positiven Befunden zu den Wirkungen von Forschendem Lernen finden: So können positive Effekte in der selbsteingeschätzten Forschungsfähigkeit (Kardash, 2000; Taraban \& Logue, 2012) oder beim Forschungsinteresse festgestellt werden (Gess, Rueß \& Deicke, 2014). Während sich das Forschungsinteresse auf die Durchführung von Forschungstätigkeiten bezieht, finden sich ebenfalls positive Entwicklungen im Hinblick auf das Interesse an der Fachdisziplin, an der Möglichkeit einer wissenschaftlichen Karriere oder an Forschendem Lernen im Allgemeinen (Seymour et al., 2004; Ward, Clarke \& Horton, 2014). Des Weiteren deuten Studien darauf hin, dass Schlüsselkompetenzen wie Kommunikations- und Teamfähigkeit durch das Forschende Lernen gefördert werden (Bauer \& Bennett, 2003; Kardash, 2000; Seymour et al., 2004; Taraban \& Logue, 2012; Ward, Clarke \& Horton, 2014). Im Hinblick auf motivationale Aspekte liegen Erkenntnisse dazu vor, dass Forschendes Lernen die intrinsische Motivation fördern kann (Seymour et al., 2004), eine hohe intrinsische Motivation zugleich jedoch eine notwendige Voraussetzung für das stark selbstbestimmungsorientierte Format darstellt (Hänze \& Moeglin, 2004; BAK, 1970; Reinmann, 2011). Befunde weisen außerdem darauf hin, dass die Wirkung von Forschendem Lernen durch motivationale Aspekte beeinflusst wird: So wurde festgestellt, dass die Leistung und die Einstellung zum selbstständigkeitsorientierten Lernen von der Ausprägung der intrinsischen Motivation abhängt (Hänze \& Moeglin, 2004) und ein Zusammenhang zwischen der intrinsischen Motivation und dem Zuwachs des Forschungsinteresses besteht (Gess, Rueß \& Deicke, 2014).

Bei bisher vorliegenden Wirkungsstudien werden jedoch keine Kontrollgruppen zur Absicherung der Wirkung berücksichtigt und es ist von Selektionseffekten auszugehen, da häufig Lehrveranstaltungen oder Programme untersucht werden, an denen Freiwillige oder nur ausgewählte Studierende teilnehmen. Weiterhin fehlen Studien, die dezidiert die Wechselwirkung motivationaler Ausgangslagen in der Wirkung berücksichtigen. Vor diesem Hintergrund gehen wir in unserem Beitrag zwei Forschungsfragen nach:

1. Welche Wirkungen hat Forschendes Lernen im Hinblick auf Forschungskompetenz? Hier erwarten wir, dass auch im Vergleich mit einer Kontrollgruppe Forschendes Lernen positive Effekte auf die Forschungskompetenz aufweist (H1).

2. Welche Rolle spielt die Motivation für die Wirkung Forschenden Lernens? Hier erwarten wir, dass Forschendes Lernen die Motivation stärker fördert als andere didaktische Formate (H2) und dass darüber hinaus motivationale Variablen mit den Wirkungen von Forschendem Lernen in Zusammenhang stehen (H3). 


\section{Forschendes Lernen und Forschungskompetenz}

Die Forschungskompetenz als Befähigung zum eigenständigen Forschen unterscheiden wir (in Anlehnung an Gess, Deicke \& Wessels, 2017) in zwei Facetten: In der kognitiven Facette betrachten wir das Wissen um Methoden inklusive des Wissens um die methodologischen Hintergründe sowie das Forschungsprozesswissen, das heißt das theoretische Wissen, wie Forschung ablaufen muss. Zusätzlich berücksichtigen wir hier die affektiv-motivationale Komponente der Forschungskompetenz, die erst dazu beiträgt, dass vorhandenes Wissen der kognitiven Komponente bei einer potenziellen Forschungstätigkeit zur Anwendung kommt (Weinert, 2001). Zu dieser affektiv-motivationalen Facette gehören neben einer allgemeinen Unsicherheitsund Komplexitätstoleranz unter anderem das gefühlsbezogene und wertbezogene Forschungsinteresse sowie die forschungsbezogene Selbstwirksamkeit (Wessels et al., 2018a, 2018b). Während sich das gefühlsbezogene Forschungsinteresse auf eine Assoziation von Forschung mit positiven Gefühlen bezieht, umfasst das wertbezogene Forschungsinteresse Überzeugungen zum Nutzen von Forschung. Bei der forschungsbezogenen Selbstwirksamkeit geht es um die subjektive Gewissheit, neue oder schwierige Forschungssituationen bewältigen zu können.

Ausgehend von diesen Überlegungen formulieren wir für unsere Studie die folgenden Hypothesen:

H1 Forschendes Lernen fördert Forschungskompetenz sowohl in der kognitiven als auch in der affektiv-motivationalen Facette stärker als andere didaktische Formate. Da die affektiv-motivationale Facette aus mehreren Dimensionen besteht, beziehen sich unsere Hypothesen konkret auf die Erwartung ...

- $\quad$ einer stärkeren Erhöhung der kognitiven Forschungskompetenz (H1_1).

- einer stärkeren Erhöhung der forschungsbezogenen Selbstwirksamkeit (H1_2).

- $\quad$ einer stärkeren Steigerung des wertbezogenen Interesses (H1_3).

\section{Forschendes Lernen und Motivation}

Bei der Betrachtung der Motivation orientieren wir uns an der Lernmotivation im Rahmen der Selbstbestimmungstheorie von Ryan und Deci, bei der Stärke und Qualität der Motivation unterschieden werden. Primär wird in dieser Theorie die Motivation im Hinblick auf das Ausmaß der Selbstbestimmung (oder Autonomie) betrachtet, wobei eine Handlung umso selbstbestimmter ist, je weniger sie 
von anderen Personen oder intrapsychischen Zwängen beeinflusst sind (Ryan \& Deci, 2000). Unterschieden wird zwischen der intrinsischen Motivation, die auf der inhärenten Befriedigung des Handlungsvollzugs beruht, und vier Formen der extrinsischen Motivation, wobei die unterste Stufe die völlig fremdbestimmte Form darstellt und die drei anderen Stufen unterschiedliche Ausprägungsgrade einer zunehmend selbstbestimmten extrinsischen Motivation beschreiben (Krapp \& Ryan, 2002, S. 61). Die intrinsische Motivation sowie die internalisierte Ausprägungsform gelten dabei als autonome Motivation, während die fremdbestimmteren Formen als kontrollierte Motivation bezeichnet werden (Ryan \& Deci, 2000).

In Rahmen der Theorie werden positive Zusammenhänge zwischen der Ausprägung der autonomen Motivation und der Lernleistung erwartet. Es wird davon ausgegangen, dass didaktische Formate, in denen das Kompetenzerleben, die Autonomie und eine soziale Verbundenheit angesprochen werden, positiv zur Entwicklung der autonomen Motivation beitragen. Weiterhin gibt es Hinweise darauf, dass Forschendes Lernen nicht nur die allgemeine Lernmotivation erhöht, sondern auch mit einer Steigerung des allgemeinen Interesses für Forschung im Studium einhergeht - im Sinne einer höheren Motivation für Forschung (Ward, Clarke \& Horton, 2014).

Wir formulieren damit in unserer Studie folgende Hypothesen:

H2 Forschendes Lernen als Lehr-Lern-Form fördert die Motivation stärker als andere didaktische Formate. Hier erwarten wir,

- dass - bezogen auf die allgemeine Lernmotivation - Forschendes Lernen die autonome Motivation stärker fördert als andere Formate (H2_1).

- dass Forschendes Lernen die allgemeine Motivation für Forschung im Studium erhöht (H2_2).

H3 Motivationale Variablen stehen in Zusammenhang mit den Wirkungen Forschenden Lernens. Hier erwarten wir,

- dass sich die Wirkungen umso stärker entwickeln, je höher die autonome Motivation zu Semesterbeginn ausgeprägt ist (H3_1).

- dass sich die Wirkungen umso stärker entwickeln, je höher die Motivation für Forschung im Studium zu Semesterbeginn ausgeprägt ist (H3_2). 


\section{$4 \quad$ Methodisches Vorgehen}

Zur Überprüfung der Hypothesen wurde ein quasi-experimentelles Prä-Post-Kontrollgruppendesign gewählt. Um im Vergleichszeitraum Veränderungen in der Forschungskompetenz und in der Motivation auf das didaktische Format des Forschenden Lernens zurückführen zu können, wurden für den Vergleich zwei inhaltlich äquivalente Module zur Einführung in die quantitativen Forschungsmethoden ausgewählt. In der Kontrollgruppe wurden die Inhalte eher „klassisch“ über eine Vorlesung mit einer Übung ohne Anteile von Forschendem Lernen vermittelt. In der Vergleichsgruppe wurden die gleichen Inhalte ebenfalls in einer grundlegenden Vorlesung vorgestellt, diese Inhalte wurden jedoch von den Studierenden in Kleingruppen in einem Projektseminar direkt an einer selbstgewählten Fragestellung angewendet und Ergebnisse aus dieser in einem Projektbericht als Prüfungsleistung dargestellt. Da das Modul nur ein Semester umfasste, war eine eigene Erhebung im Seminarkontext nicht möglich; die Fragen wurden - wie in der quantitativen Forschung sehr häufig - sekundäranalytisch an einem thematisch sehr umfangreichen Datensatz bearbeitet.

Die ausgewählten Module sind jeweils Pflichtmodule für Studierende des ersten Semesters im Bachelor-Studiengang Erziehungswissenschaft (Kontrollgruppe) bzw. Pädagogik (Forschendes-Lernen-Gruppe). Wir beziehen somit in unsere Studie explizit Studierende ein, die a) noch keine bzw. kaum forschungsmethodische Kenntnisse aufweisen und b) noch relativ wenig Erfahrung mit Forschung haben.

In beiden Modulen wurde die Erhebung jeweils zu Beginn des Semesters in der ersten Vorlesungssitzung sowie am Ende des Semesters durchgeführt. Im „klassischen" Modul wurde zum Abschluss des Semesters eine Klausur geschrieben, das Modul im Format des Forschenden Lernens endete dagegen erst mit Abgabe des Projektberichts sechs Wochen später. Da die Fertigstellung des Projektberichtes noch einmal eine intensive Beschäftigung mit den Modulinhalten notwendig macht, hätte für eine Vergleichbarkeit der beiden Module die Post-Erhebung in der Forschendes-Lernen-Gruppe später durchgeführt werden müssen als in der Kontrollgruppe. Aus organisatorischen Gründen musste hiervon jedoch abgesehen werden.

\subsection{Beschreibung der Stichprobe}

Insgesamt liegen von 92 Personen Daten für beide Erhebungszeitpunkte vor. Zum ersten Messzeitpunkt in der ersten Vorlesungssitzung haben 191 Studierende den Fragebogen ausgefüllt, in der letzten Sitzung 137 Studierende. Die Teilstichproben sind sich relativ ähnlich (siehe Tab. 1) und weisen nur leichte - nicht signifikante 
- Unterschiede auf. So finden sich in der Teilstichprobe der Forschendes-Lernen-Gruppe mehr Studierende im ersten Fachsemester und weniger Studierende mit Abitur als Hochschulzugangsberechtigung, dafür sind diese weniger häufig neben dem Studium erwerbstätig.

Tab. 1 Stichprobenbeschreibung

\begin{tabular}{lll}
\hline & FL & KG \\
\hline Fälle $(n)$ & 52 & 40 \\
Alter $M(S D)$ & $24,2(6,4)$ & $24,6(7,2)$ \\
Anteil 1. Semester & $88,5 \%$ & $75 \%$ \\
Geschlecht (weiblich) & $80,8 \%$ & $74,4 \%$ \\
Kinder (ja) & $11,5 \%$ & $10,0 \%$ \\
Studienberechtigung (Abitur) & $84,3 \%$ & $94,9 \%$ \\
Berufstätigkeit neben Studium (ja) & $47,1 \%$ & $59,0 \%$ \\
\hline
\end{tabular}

Anmerkungen: Anteil 1. Semester auf Basis des 2. Messzeitpunkts. Alle übrigen Angaben beziehen sich auf den 1. Messzeitpunkt. Keine signifikanten Unterschiede zwischen FL und $\mathrm{KG}$.

\subsection{Erhebungsinstrumente}

\subsubsection{Forschungskompetenz}

Zur Erfassung der Forschungskompetenz haben wir einen Teil eines erprobten Instrumentes zur Messung der Forschungskompetenz genutzt (Gess, Geiger \& Ziegler, 2018). Wir haben in unserer Studie aus diesem Kompetenztest die $18 \mathrm{Mul}-$ tiple-Choice-Fragen zu quantitativen Forschungsmethoden sowie zum Forschungsprozesswissen eingesetzt. Obwohl wir hier ein etabliertes Instrument mit guten Gütekriterien bei Studierenden ab dem dritten Fachsemester ausgewählt haben, scheint der Test für unsere Stichprobe mit Studierenden des ersten Semesters weniger passend zu sein: Wir erhalten in unseren Stichproben keine zufriedenstellenden Reliabilitäten (Reliabilität in $\mathrm{t}_{1} \omega_{\mathrm{t}}=.60$, in $\mathrm{t}_{2} \omega_{\mathrm{t}}=.64$ ) und mussten den Test aufgrund geringer Trennschärfen in einzelnen Items auf insgesamt 15 Items reduzieren.

Die affektiv-motivationale Komponente der Forschungskompetenz wird in unserer Studie durch die beiden Aspekte der forschungsbezogenen Selbstwirksamkeit (neun Items umfassende Skala) und des wertbezogenen Interesses (sechs Items umfassende Skala) berücksichtigt. Für die forschungsbezogene Selbstwirksamkeit sind 
die Reliabilitäten zu $t 1$ und $t 2$ ausreichend, die Skala zum wertbezogenen Interesse erreicht jedoch zu t1 keine ausreichende Reliabilität (siehe Tab. 2). ${ }^{1}$

Tab. 2 Instrumente zur Erfassung der affektiv-motivationalen Forschungskompetenz mit Beispiel-Item

\begin{tabular}{|c|c|c|c|}
\hline Konstrukt & Beispiel-Item & $\begin{array}{c}\mathrm{N} \\
\text { Items }\end{array}$ & $\alpha\left(t_{1} / t_{2}\right)$ \\
\hline $\begin{array}{l}\text { Forschungsbezogene } \\
\text { Selbstwirksamkeit } \\
\text { (Wessels, Rueß \& Gess, 2019) }\end{array}$ & $\begin{array}{l}\text { "Ich bin mir sicher, dass ich Metho- } \\
\text { den zur Datenerhebung anwenden } \\
\text { kann, auch wenn ich diese vorher } \\
\text { noch nicht benutzt habe." }\end{array}$ & 9 & $.74 / .72$ \\
\hline $\begin{array}{l}\text { Wertbezogenes Interesse } \\
\text { an Forschung } \\
\text { (Wessels, Rueß \& Gess, 2018) }\end{array}$ & $\begin{array}{l}\text { „Nur durch Forschung kann in } \\
\text { meinem Fach das bestehende Wis- } \\
\text { sen erweitert werden“ }\end{array}$ & 6 & $.53 / .73$ \\
\hline
\end{tabular}

\subsubsection{Motivation}

Die Motivation für Forschung im Studium wird über eine selbstentwickelte Skala (sechs Items) erhoben, mit der abgebildet wird, in welchem Ausmaß Forschung im Studium angestrebt wird. Die Skala zur autonomen Motivation umfasst acht Items und orientiert sich an Formulierungen der Skalen zur motivationalen Regulation beim Lernen (Müller, Hanfstingl \& Andreitz, 2007). Die ursprünglich für Schülerinnen und Schüler gedachten Skalen wurden auf Studierende angepasst und die beiden Skalen zur intrinsischen und internalisierten Motivation zu einer Skala der autonomen Motivation verrechnet. Die Reliabilitäten der beiden Instrumente zur Erfassung der Motivation weisen knapp zufriedenstellende Reliabilitäten auf (siehe Tab. 3).

1 Das gefühlsbezogene Interesse (sowie weitere affektiv-motivationale Variablen) der Forschungskompetenz haben wir nicht berücksichtigt, weil sich diese Konstrukte nicht valide auf Studienanfängerinnen und Studienanfänger übertragen lassen. Für das wertbezogene Interesse müssen wir angesichts der geringen Reliabilität zu t1 ebenfalls in Erwägung ziehen, dass dieses für die von uns untersuchte Zielgruppe nicht perfekt geeignet ist. 
Tab. 3 Instrumente zur Erfassung der Motivation mit Beispiel-Item

\begin{tabular}{llcc}
\hline Konstrukt & Beispiel-Item & $\begin{array}{c}\mathbf{N} \\
\text { Items }\end{array}$ & $\boldsymbol{\alpha}\left(\mathbf{t}_{\mathbf{1}} / \mathbf{t}_{\mathbf{2}}\right)$ \\
\hline $\begin{array}{l}\text { Motivation für } \\
\text { Forschung im Studium }\end{array}$ & $\begin{array}{l}\text { "Je forschungsorientierter mein Studium } \\
\text { umso besser“ }\end{array}$ & $.68 / .84$ \\
Autonome Motivation & $\begin{array}{l}\text { „Ich lerne im Studium, weil ich es genieße, } \\
\text { mich mit meinem Studienfach auseinan- } \\
\text { derzusetzen“ }\end{array}$ & 8 & $.71 / .65$ \\
\hline
\end{tabular}

\subsection{Datenanalyse}

Die Hypothesen zu den Wirkungen von Forschendem Lernen (H1 und H2) werden in zwei Schritten überprüft.

1. Für jede Gruppe (Interventions- und Kontrollgruppe) werden für die Wirkungsvariablen t-Tests für abhängige Stichproben inklusive der Effektstärken berechnet. So kann festgestellt werden, ob - für jede Gruppe getrennt betrachtet - signifikante Entwicklungen in den abhängigen Variablen vorliegen und wie stark diese Entwicklungen ausgefallen sind. Wenn sich die Variablen der Forschungskompetenz und der Motivation im Semesterverlauf durch die Teilnahme an den Veranstaltungen verändert haben, sollte der Test ein signifikantes Ergebnis liefern. Falls die Veranstaltungen zu einer Entwicklung beitragen, ist es darüber hinaus interessant, zu betrachten, wie stark diese Entwicklung ist. Zur Interpretation der Stärke nutzen wir die Effektstärke $\mathrm{d}$ : $\mathrm{d}=.2$ weist auf einen kleinen Effekt, $\mathrm{d}=.5$ auf einen mittleren Effekt und $\mathrm{d}=.8$ auf einen großen Effekt hin (Cohen, 1988).

2. Um Unterschiede in den Entwicklungen auf das didaktische Format zurückführen zu können, werden zusätzlich zweifaktorielle Varianzanalysen mit Messwiederholung berechnet. Bei einer unterschiedlichen Entwicklung wird gemäß der Hypothesen erwartet, dass ein signifikanter Interaktionseffekt zwischen den unabhängigen Variablen „Messzeitpunkt" und „didaktisches Format"vorliegt. Bei Vorliegen eines solchen Interaktionseffektes wird die Bedeutsamkeit des Effektes durch das Effektstärkenmaß partielles Eta-Quadrat $\left(\eta p^{2}\right)$ ausgedrückt. Dieses Effektstärkenmaß stellt zwar für den Vergleich unterschiedlicher Studien ein ungünstiges Maß dar (Rasch et al., 2014), da sich aber bisher noch kein alternatives Maß für messwiederholte Designs durchgesetzt hat, wird es hier trotzdem verwendet. Eine grobe Orientierung für die Interpretation können hier die Faustregeln von Cohen für Eta-Quadrat bei unabhängigen Designs 
liefern: $\eta^{2}=0.01$ kleiner Effekt, $\eta^{2}=0.06$ mittlerer Effekt und $\eta^{2} \geq 0.14$ großer Effekt Cohen, 1988).

Zur Prüfung der Hypothese 3 werden Differenzwerte für die abhängigen Wirkungsvariablen gebildet und diese mit der autonomen Motivation zu Beginn des Semesters ( $\mathrm{t} 1$ ) beziehungsweise mit der Ausprägung der Motivation für Forschung zu Beginn des Semesters (t1) korreliert (Pearson Korrelation). Erwartet werden positive Korrelationen.

\section{$5 \quad$ Ergebnisse}

\subsection{Ergebnisse zur Forschungskompetenz (H1)}

Für die kognitive Forschungskompetenz können wir signifikante Verbesserungen in beiden Stichproben feststellen; sie weisen eine mittlere Effektstärke auf (siehe Tab. 4). Da die inhaltliche Ausrichtung beider Module stark auf der kognitiven Forschungskompetenz lag, ist dieser Befund nicht überraschend. Zwar ist der Effekt der Veränderungen in der Forschendes-Lernen-Stichprobe mit d=.66 etwas höher als in der Kontrollgruppe $(\mathrm{d}=.62)$, eine unterschiedliche Entwicklung der beiden Stichproben können wir allerdings varianzanalytisch nicht feststellen. Eine stärkere Förderung der kognitiven Forschungskompetenz durch das Format Forschenden Lernens ist somit nicht zu erkennen.

Im Hinblick auf die forschungsbezogene Selbstwirksamkeit können keine Entwicklungen beobachtet werden. Die Veränderungen der Mittelwerte $(\Delta M)$ im Semesterverlauf tendieren gegen null. Beide didaktischen Ansätze tragen somit nicht zur Entwicklung forschungsbezogener Selbstwirksamkeit bei. Auch in Bezug auf die Förderung des wertbezogenen Interesses an Forschung lassen sich im Semesterverlauf keine signifikanten Veränderungen in den beiden Gruppen erkennen. Auf der deskriptiven Ebene lässt sich für die Forschendes-Lernen-Gruppe sogar eine geringe - jedoch nicht signifikante - Abnahme des wertbezogenen Interesses an Forschung beobachten $(\Delta \mathrm{M}=-.13)$. 
Tab. 4 Ergebnisse zur Hypothese 1

\begin{tabular}{|c|c|c|c|c|c|c|c|c|}
\hline & $\mathrm{N}$ & $M(S D)_{t 1}$ & $M(S D)_{t 2}$ & $\mathrm{M} \Delta$ & $\mathrm{t}(\mathrm{d})$ & $\begin{array}{c}F_{t} \\
\left(\eta^{2}\right)\end{array}$ & $F_{t^{\star} F L_{\overline{2}} K G}$ & $\begin{array}{c}F_{F L K G} \\
\left(\eta^{2}\right)\end{array}$ \\
\hline \multicolumn{9}{|c|}{$\begin{array}{l}\text { H1_1 } \\
\text { Kognitive Forschungskompetenz }\end{array}$} \\
\hline $\begin{array}{l}\text { FL } \\
\text { KG }\end{array}$ & $\begin{array}{l}51 \\
40\end{array}$ & $\begin{array}{l}8.02(2.41) \\
7.15(2.75)\end{array}$ & $\begin{array}{l}9.64(2.47) \\
8.83(2.63)\end{array}$ & $\begin{array}{l}1.62 \\
1.68 \\
\end{array}$ & $\begin{array}{l}4.88^{\star \star}(0.66) \\
4.37^{\star \star}(0.62)\end{array}$ & $\begin{array}{c}42.44^{\star *} \\
(.32)\end{array}$ & $\begin{array}{l}0.14 \\
(.00) \\
\end{array}$ & $\begin{array}{l}3.14 \\
(.03)\end{array}$ \\
\hline \multicolumn{9}{|c|}{$\begin{array}{l}\text { H1_2 } \\
\text { Forschungsbezogene Selbstwirksamkeit }\end{array}$} \\
\hline $\begin{array}{l}\text { FL } \\
\text { KG }\end{array}$ & $\begin{array}{l}51 \\
38\end{array}$ & $\begin{array}{l}3.27(.48) \\
3.36(.45)\end{array}$ & $\begin{array}{l}3.20(.47) \\
3.32(.53)\end{array}$ & $\begin{array}{l}-0.06 \\
-0.04\end{array}$ & $\begin{array}{l}0.76(0.13) \\
0.49(0.08)\end{array}$ & $\begin{array}{l}0.74 \\
(.01)\end{array}$ & $\begin{array}{l}0.04 \\
(.00)\end{array}$ & $\begin{array}{l}1.39 \\
(.02)\end{array}$ \\
\hline \multicolumn{9}{|c|}{$\begin{array}{l}\text { H1_3 } \\
\text { Wertbez. Interesse an Forschung }\end{array}$} \\
\hline $\begin{array}{l}\text { FL } \\
\text { KG }\end{array}$ & $\begin{array}{l}51 \\
40\end{array}$ & $\begin{array}{l}3.67(.46) \\
3.74(.46)\end{array}$ & $\begin{array}{l}3.54(.55) \\
3.70(.58)\end{array}$ & $\begin{array}{l}-0.13 \\
-0.04\end{array}$ & $\begin{array}{l}1.60(0.25) \\
0.49(0.08)\end{array}$ & $\begin{array}{l}2.07 \\
(.02)\end{array}$ & $\begin{array}{l}0.52 \\
(.01)\end{array}$ & $\begin{array}{l}1.61 \\
(.02)\end{array}$ \\
\hline
\end{tabular}

\subsection{Ergebnisse zur Motivation (H2 und H3)}

Beide Gruppen weisen zu Semesterende eine geringere autonome Motivation auf als zu Semesterbeginn, wobei diese Abnahme nur für die Kontrollgruppe signifikant ist (siehe Tab. 5). Eine unterschiedliche Entwicklung der autonomen Motivation kann varianzanalytisch jedoch nicht festgestellt werden, sodass wir davon ausgehen müssen, dass beide didaktischen Formate gleichermaßen eher zu einer Abnahme der autonomen Motivation beitragen. Eine signifikant unterschiedliche Entwicklung in den beiden didaktischen Formaten kann dagegen für die Motivation an Forschung im Studium festgehalten werden: Die Studierenden der Forschendes-Lernen-Gruppe weisen am Ende des Semesters eine signifikant geringere Motivation für Forschung

Tab. 5 Ergebnisse zur Hypothese 2

\begin{tabular}{|c|c|c|c|c|c|c|c|c|}
\hline & $\mathrm{N}$ & $M(S D)_{t 1}$ & $M(S D)_{t 2}$ & $\mathrm{M} \Delta$ & $\mathrm{t}(\mathrm{d})$ & $F_{t}\left(\eta^{2}\right)$ & 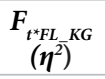 & $\begin{array}{c}F_{F L_{-} K G} \\
\left(\eta^{2}\right)\end{array}$ \\
\hline \multicolumn{9}{|c|}{ H2_1 Autonome Motivation } \\
\hline $\begin{array}{l}\text { FL } \\
\text { KG }\end{array}$ & $\begin{array}{l}52 \\
39\end{array}$ & $\begin{array}{l}4.10(.48) \\
4.00(.47)\end{array}$ & $\begin{array}{l}3.97(.43) \\
3.81(.48)\end{array}$ & $\begin{array}{l}-.12 \\
-.19\end{array}$ & $\begin{array}{c}1.9(.26) \\
2.56^{*}(.41)\end{array}$ & $\begin{array}{c}10.40^{\star *} \\
(.11)\end{array}$ & $\begin{array}{l}.63 \\
(.01)\end{array}$ & $\begin{array}{l}1.86 \\
(.02)\end{array}$ \\
\hline \multicolumn{9}{|c|}{ H2_2 Motivation für Forschung im Studium } \\
\hline $\begin{array}{l}\text { FL } \\
\text { KG }\end{array}$ & $\begin{array}{l}52 \\
40\end{array}$ & $\begin{array}{l}3.12(.50) \\
3.35(.59)\end{array}$ & $\begin{array}{l}2.99(.76) \\
3.40(.61)\end{array}$ & $\begin{array}{l}-.13 \\
.05\end{array}$ & $\begin{array}{c}2.15^{\star}(.29) \\
-.70(.08)\end{array}$ & $\begin{array}{l}1.44 \\
(.02)\end{array}$ & $\begin{array}{l}\mathbf{4 . 0 8}^{\star} \\
(.04)\end{array}$ & $\begin{array}{l}5.87^{\star} \\
(.06)\end{array}$ \\
\hline
\end{tabular}


im Studium auf als zu Semesterbeginn. Im varianzanalytischen Vergleich unterscheiden sich beide Gruppen einerseits generell in ihrer Motivation für Forschung $\left(\mathrm{F}_{\mathrm{FL} \_\mathrm{KG}}\right)$, andererseits entwickelt sich ihre Motivation im Semesterverlauf signifikant unterschiedlich $\left(\mathrm{F}_{t^{*} F_{L} \_K G}\right)$ : Wir können hier somit von einem insgesamt geringeren Niveau von Motivation für Forschung in der Forschendes-Lernen-Gruppe und von einer unterschiedlichen Wirkung beider didaktischen Formate ausgehen - wobei die Ergebnisse konträr zu unseren Annahmen auf eine Abnahme der Motivation für Forschung durch Forschendes Lernen hinweisen.

Entgegen unseren Annahmen in Hypothese 3 steht weder die anfängliche Ausprägung der autonomen Motivation noch die Motivation für Forschung in einem signifikanten Zusammenhang mit der Entwicklung unserer ausgewählten Wirkungsvariablen (siehe. Tab. 6).

Tab. 6 Ergebnisse zu Hypothese 3

\begin{tabular}{lcc}
\hline Veränderung in: & $\begin{array}{c}\text { Autonome } \\
\text { Motivation zu t1 }\end{array}$ & $\begin{array}{c}\text { Motivation für } \\
\text { Forschung zu t1 }\end{array}$ \\
\hline Kognitive Forschungskompetenz & -.17 & .13 \\
\hline Forschungsbezogene Selbstwirksamkeit & -.09 & .00 \\
\hline Wertbez. Interesse an Forschung & -.05 & .01 \\
\hline Motivation für Forschung im Studium & -.11 & -- \\
\hline Autonome Motivation & -- & -.07 \\
\hline
\end{tabular}

\section{Diskussion}

Zusammengefasst sind die Befunde zur Wirkung von Forschendem Lernen ernüchternd: Im Kontrollgruppenvergleich sind weder positive Effekte in den ausgewählten Facetten der Forschungskompetenz noch im Hinblick auf die motivationalen Variablen erkennbar. Die kognitive Forschungskompetenz verbessert sich (unabhängig vom didaktischen Format) in beiden Gruppen und die beiden affektiv-motivationalen Facetten der Forschungskompetenz zeigen in beiden Gruppen keine Veränderungen über den Semesterverlauf. Auch einen positiven Effekt auf die Motivation können wir nicht nachweisen: Während die allgemeine autonome Lernmotivation mit Semesterverlauf abnimmt, reduziert sich die Motivation für Forschung im Studium sogar in der Forschendes-Lernen-Gruppe. Damit scheint das (aufwendigere) Format des Forschenden Lernens die kognitive und die affektiv-motivationale Facetten der 
Forschungskompetenz sowie allgemeine motivationale Variablen nicht stärker zu fördern als klassische didaktische Formate - oder sogar negativ zu beeinträchtigen, wie für das Konstrukt der Motivation für Forschung im Studium erkennbar.

Für diese Befunde sind einige Erklärungen möglich:

1. In beiden untersuchten Gruppen scheint der Fokus sehr stark auf der Wissensvermittlung im Feld der Statistik und somit auf der kognitiven Facette von Forschungskompetenz zu liegen. Motivation und Affekt für Forschung oder das Studium allgemein werden womöglich in beiden Formaten nicht explizit gefördert. Die fehlenden Effekte könnten demnach mit der hier untersuchten spezifischen Form von Forschendem Lernen zusammenhängen und können nicht ohne Weiteres auf Formate übertragen werden, die stärker dem idealtypischen Ablauf von Forschendem Lernen entsprechen (Rueß, Gess \& Deicke, 2016). Ein Indiz hierfür könnten die - auch im Vergleich zu anderen Studien - hohen Effektstärken der Veränderung in der kognitiven Forschungskompetenz sein. So konnte für andere Formen von Forschendem Lernen, die den Forschungsprozess stärker in den Vordergrund rücken, nur eine kleine Effektstärke von $\mathrm{d}=.24$ ermittelt werden (Wessels et al., 2017). Ein weiteres Indiz ergibt sich aus dem Befund, dass die Veränderung des Forschungsinteresses im Zusammenhang steht zu Art und Anzahl der absolvierten Forschungsschritte: Insbesondere die Bearbeitung von Forschungsliteratur, die Entwicklung von Forschungsdesigns und die Datenerhebung zeigten einen positiven Zusammenhang zum Entwicklung des Forschungsinteresses (Gess, Rueß \& Deicke, 2014).

2. In dem in unserer Studie untersuchten Beispielmodul für Forschendes Lernen wird der Forschungsprozess sehr stark angeleitet vorgenommen. Angenommen wurde, dass diese Struktur in der erstmaligen Durchführung eines Forschungsprozesses dazu führt, die forschungsbezogene Selbstwirksamkeit zu fördern. Stattdessen müssen wir feststellen, dass auch in der Forschendes-Lernen-Gruppe keine Veränderungen in der forschungsbezogenen Selbstwirksamkeit stattgefunden haben. Eine mögliche Erklärung hierfür könnte sein, dass sich bei den Studierenden zeitgleich eine realistischere Einschätzung der Herausforderungen des Forschungsprozesses ausbildet, die der Förderung der forschungsbezogenen Selbstwirksamkeit entgegenwirkt. Auch die Abnahme der Motivation für Forschung im Studium könnte mit einer eher realistischeren Einschätzung des Aufwandes für Forschung zusammenhängen. Im Umkehrschluss bedeutete diese Erklärung jedoch auch, dass sich die Testwerte der genutzten Instrumente zu Semesterbeginn aufgrund des Mangels an Forschungserfahrungen nicht valide interpretieren lassen. Dies könnte in Folgestudien untersucht werden, indem retrospektive Pre-Messungen angewendet werden, bei denen Studierende 
zu Semesterende über ihre Einstellungen bei Semesterbeginn befragt werden (Coulter, 2012; Lam \& Bengo, 2003)

3. Die fehlenden positiven Effekte könnten weiterhin mit zwei Besonderheiten der Stichproben zusammenhängen: Erstens wurden Studienanfängerinnen und -anfänger untersucht, zweitens fand die Teilnahme an den untersuchten Veranstaltungen verpflichtend statt. Es ist zu vermuten, dass zumindest jeweils ein Teil der Studierenden durch Forschendes Lernen überfordert wird oder wenig forschungsaffin ist. Bei freiwilliger Teilnahme konnte nämlich eine positive Entwicklung des Forschungsinteresses beobachtet werden (Gess, Rueß \& Deicke, 2014). Dies wirft zwei Fragen auf: (1) Ist Forschendes Lernen zum Studienbeginn geeignet? Diese Frage ließe sich beantworten, indem unser Forschungsdesign auf Veranstaltungen beispielsweise im dritten Bachelorsemester angewendet wird (reine Vorlesung vs. Lehrforschungsprojekte). (2) Ist Forschendes Lernen unter Zwangsbedingungen für forschungsdistanzierte Studierende kontraproduktiv? Um dieser Frage nachzugehen, müsste Forschendes Lernen möglichst durch dieselbe Lehrkraft sowohl in einem Pflichtmodul als auch in einem Wahlpflichtbereich angeboten werden.

4. In der vorliegenden Studie sind nur sehr kleine Stichproben und weder weitere Lehrveranstaltungsmerkmale noch Merkmale der Lehrenden berücksichtigt. Dabei können die Kursbedingungen insbesondere die affektiv-motivationalen Variablen beeinflussen. Die Wirkungen von Forschendem Lernen könnten in besonderer Weise damit verbunden sein, dass Lehrende Studierenden Spaß an der Forschung vermitteln oder zumindest vorleben (Rueß, Wessels \& Deicke, 2019). In zukünftigen Studien wäre eine Ausweitung auf mehr Studierende und die Berücksichtigung weiterer Einflussvariablen wünschenswert.

Insgesamt lassen die Ergebnisse den vorsichtigen Schluss zu, dass der aufwendige Einsatz von Forschendem Lernen zumindest bei Studienneulingen sowie im Rahmen von Pflichtveranstaltungen fragwürdig ist. Sollte sich dieser Befund auch in anderen Kontexten und bei Verwendung anderer Instrumente, idealerweise in Kombination mit retrospektiver Pre-Messung, replizieren, muss die vermutete Wirkung von Forschendem Lernen auf Forschungskompetenz hinterfragt werden. In Bezug auf die autonome Motivation kann festgehalten werden, dass zumindest im hier untersuchten Setting eine hoch ausgeprägte autonome Motivation für die Entwicklung der anvisierten Kompetenzen nicht förderlich ist. Damit stehen unsere Befunde im Widerspruch zu eingangs erwähnten positiven Zusammenhängen (u. a. Gess et al., 2014; Kardash, 2000; Seymour et al., 2004; Taraban \& Logue, 2012). 


\section{Literatur}

Bundesassistentenkonferenz (BAK) (1970). Forschendes Lernen - Wissenschaftliches Prüfen (Schriften der BAK, 5. Neudruck) Bielefeld: UVW.

Bauer, K. W. \& Bennett, J. S. (2003). Alumni Perceptions Used to Assess Undergraduate Research Experience. The Journal of Higher Education, 74(2), S. 210-230.

Cohen, J. (1988). Statistical power analysis for the behavioral sciences. Hillsdale: Erlbaum.

Coulter, S. E. (2012). Using the retrospective pretest to get usable, indirect evidence of student learning. Assessment \& Evaluation in Higher Education, 37(3), S. 321-334.

Gess, C., Rueß, J. \& Deicke, W. (2014). Design-based Research als Ansatz zur Verbesserung der Lehre an Hochschulen: Einführung und Praxisbeispiel. Qualität in der Wissenschaft, $8(1)$, S. 10-16.

Gess, C., Deicke, W. \& Wessels, I. (2017). Kompetenzentwicklung durch Forschendes Lernen. In H. Mieg \& J. Lehmann (Hrsg.), Forschendes Lernen: Wie die Lehre in Universität und Fachhochschule erneuert werden kann (S.79-90). Frankfurt: Campus.

Gess, C., Geiger, C. \& Ziegler, M. (16.02.2018). Social-Scientific Research Competency: Validation of Test Score Interpretations for Evaluative Purposes in Higher Education. European Journal of Psychological Assessment. Verfügbar unter https://econtent.hogrefe. com/doi/pdf/10.1027/1015-5759/a000451 [28.03.2018].

Hänze, M. \& Moegling, K. (2004). Forschendes Lernen als selbstständigkeitsorientierte Unterrichtsform: Persönliche Voraussetzungen und motivationale Wirkmechanismen. Psychologie in Erziehung und Unterricht, 51, S. 113-115.

Huber, L. (2004). Forschendes Lernen: 10 Thesen zum Verhältnis von Forschung und Lehre aus der Perspektive des Studiums. Die Hochschule, 13(2): S. 29-49.

Huber, L. (2009). Warum forschendes Lernen nötig und möglich ist. In L. Huber, J. Hellmer \& F. Schneider (Hrsg.), Forschendes Lernen im Studium: Aktuelle Konzepte und Erfahrungen (S.9-35). Bielefeld: UVW.

Kardash, C. M. (2000). Evaluation of an undergraduate research experience: Perceptions of undergraduate interns and their faculty mentors. Journal of Educational Psychology, 92(1), S. 191-201.

Krapp, A. \& Ryan, R. M. (2002). Selbstwirksamkeit und Lernmotivation: Eine kritische Betrachtung der Theorie von Bandura aus der Sicht der Selbstbestimmungstheorie und der pädagogisch-psychologischen Interessentheorie. In M. Jerusalem \& D. Hopf (Hrsg.), Selbstwirksamkeit und Motivationsprozesse in Bildungsinstitutionen (S. 54-82). Weinheim: Beltz.

Lam, T. C. M. \& Bengo, P. (2003). A Comparison of Three Retrospective Self-reporting Methods of Measuring Change in Instructional Practice. American Journal of Evaluation, 24(1), S.65-80. Verfügbar unter https://journals.sagepub.com/doi/ pdf/10.1177/109821400302400106 [28.03.2019].

Müller, F. H., Hanfstingl, B. \& Andreitz, I. (2007). Skalen zur motivationalen Regulation beim Lernen von Schülerinnen und Schülern: Adaptierte und ergänzte Version des Academic Self-Regulation Questionnaire (SRQ-A) nach Ryan und Connell (1989) (Wissenschaftliche Beiträge aus dem Institut für Unterrichts- und Schulentwicklung Nr. 1). Klagenfurt: Alpen-Adria-Universität.

Rasch, B., Friese, M., Hofmann, W., Neumann, E. (2014). Quantitative Methoden 2: Einführung in die Statistik für Psychologen und Sozialwissenschaftler. Heidelberg: Springer. 
Reinmann, G. (2011). Forschendes Lernen und wissenschaftliches Prüfen: Die potentielle und faktische Rolle der digitalen Medien. In T. Meyer, W.-H. Tan, C. Schwalbe \& R. Appelt (Hrsg.), Medien \& Bildung (S. 291-306). Wiesbaden: VS.

Rueß, J., Gess, C. \& Deicke, W. (2016). Forschendes Lernen und forschungsbezogene Lehre - empirisch gestützte Systematisierung des Forschungsbezugs hochschulischer Lehre. Zeitschrift für Hochschulentwicklung, 11(2), S.23-44.

Rueß, J., Wessels, I. \& Deicke, W. (2019). Does research-based learning facilitate the development of research competencies? Results from a pre-post analysis in 74 university courses in the social sciences. Vortrag auf der Tagung "focus URE“, Universität Hohenheim, 05.-07.06.2019.

Ryan, R. M. \& Deci, E. L. (2000). Self-determination theory and the facilitation of intrinsic motivation, social development, and well-being. American Psychologist, 55(1), S. 68-78.

Schubarth, W. \& Speck, K. (2014). Employability und Praxisbezüge im wissenschaftlichen Studium: Fachgutachten für die Hochschulrektorenkonferenz. Verfügbar unter https:// www.hrk-nexus.de/fileadmin/redaktion/hrk-nexus/07-Downloads/07-02-Publikationen/ Fachgutachten_Employability-Praxisbezuege.pdf [28.03.2019].

Seymour, E., Hunter, A.-B., Laursen, S. L. \& Deantoni, T. (2004). Establishing the Benefits of Research Experiences for Undergraduates in the Sciences: First Findings from a ThreeYear Study. Science education, 88(4), S.493-534.

Taraban, R. \& Logue, E. (2012). Academic Factors That Affect Undergraduate Research Experiences. Journal of Educational Psychology, 104(2), S. 499-514.

Ward, J. R., Clarke, H. D. \& Horton, J. L. (2014). Effects of a Research-Infused Botanical Curriculum on Undergraduates' Content Knowledge, STEM Competencies, and Attitudes toward Plant Sciences. CBE - Life Sciences Education, 13(3), S.387-396.

Weinert, F. E. (2001). Concept of competence: A Conceptual Clarification. In D. S. Rychen \& L. H. Salganik (Hrsg.), Defining and selecting key competencies (S. 45-65). Seattle: Hogrefe.

Wessels, I. (2017). Welche Kompetenzen werden durch die Teilnahme an Forschendem Lernen erworben? - Erste Ergebnisse einer Wirkungsanalyse an 10 Universitäten. Vortrag an der Carl von Ossietzky Universität Oldenburg, 27.07.2017.

Wessels, I., Rueß, J. \& Gess, C. (2018). Instrument zur Erhebung des wertbezogenen Interesses an Forschung. Berlin: bologna.lab der Humboldt-Universität zu Berlin. Verfügbar unter https://bolognalab.hu-berlin.de/de/begleitende_forschung/material/Forschung/w-Interesse [20.02.2019].

Wessels, I., Rueß, J. \& Gess, C. (2019). Instrument zur Erhebung der Selbstwirksamkeit im Forschungsprozess. Berlin: bologna.lab der Humboldt-Universität zu Berlin.

Wessels, I., Rueß, J, Deicke, W. \& Jenßen, L. (2018a). Which competencies can be acquired through research-based learning? - Results from a pre-post analysis in 74 university courses. In H. A. Mieg, W. Deicke, F. Fischer \& J. Zottmann (Hrsg.), Learning through Inquiry in Higher Education: Current Research and Future Challenges (INHERE 2018). München. Verfügbar unter https://doi.org/10.3205/18inhere30 [09.09.2019].

Wessels, I., Rueß, J., Jenßen, L., Gess, C. \& Deicke, W. (2018b). Beyond cognition: Experts' views on affective-motivational research dispositions in the social sciences. Frontiers in Psychology, 9(1300). Verfügbar unter https://doi.org/10.3389/fpsyg.2018.01300 [09.0.9.2019].

Wissenschaftsrat (2006). Empfehlungen zur künftigen Rolle der Universitäten im Wissenschaftssystem. Berlin: Wissenschaftsrat.

Wissenschaftsrat (2015). Empfehlungen zum Verhältnis von Hochschulbildung und Arbeitsmarkt. Bielefeld: Wissenschaftsrat. 


\section{Zu den Autorinnen und zum Autor}

Dr. Carmen Wulf ist Diplom-Psychologin und war von 2011 bis 2020 Wissenschaftliche Mitarbeiterin an der Carl von Ossietzky Universität Oldenburg für das Projekt „Forschendes Lernen im Fokus“. Sie hat in dieser Zeit eine Vielzahl von Lehrveranstaltungen im Format des Forschenden Lernens angeboten und sich in der Forschung unter anderem mit Evaluationen in schulischen und hochschulischen Kontexten und mit Einstellungen Studierender zum Lernen beschäftigt. E-Mail: carmen.wulf@uol.de

Dr. Janina Thiem ist Mitarbeiterin in der Internen Evaluation im Referat Studium und Lehre der Carl von Ossietzky Universität Oldenburg und für die Projektevaluation des Qualitätspakt-Lehre-Projekts „Forschungsbasiertes Lernen im Fokus (FLiF)“ zuständig. E-Mail: janina.thiem@uol.de

Dr. Christopher Gess hat ein Lehramtsstudium an der Universität Bielefeld mit dem ersten Staatsexamen sowie ein Masterstudium der Public Administration an der Columbia University (USA) abgeschlossen. Er arbeitete von 2012 bis 2017 im bologna. lab der Humboldt-Universität zu Berlin. Dort lag sein Forschungsschwerpunkt auf den Wirkungen Forschenden Lernens, insbesondere auf dem Forschungsinteresse und der Forschungskompetenz. Letzteres ist das Thema seiner 2019 abgeschlossenen Dissertation. E-Mail: christopher.gess@hu-berlin.de

Open Access Dieses Kapitel wird unter der Creative Commons Namensnennung 4.0 International Lizenz (http://creativecommons.org/licenses/by/4.0/deed.de) veröffentlicht, welche die Nutzung, Vervielfältigung, Bearbeitung, Verbreitung und Wiedergabe in jeglichem Medium und Format erlaubt, sofern Sie den/die ursprünglichen Autor(en) und die Quelle ordnungsgemäß nennen, einen Link zur Creative Commons Lizenz beifügen und angeben, ob Änderungen vorgenommen wurden.

Die in diesem Kapitel enthaltenen Bilder und sonstiges Drittmaterial unterliegen ebenfalls der genannten Creative Commons Lizenz, sofern sich aus der Abbildungslegende nichts anderes ergibt. Sofern das betreffende Material nicht unter der genannten Creative Commons Lizenz steht und die betreffende Handlung nicht nach gesetzlichen Vorschriften erlaubt ist, ist für die oben aufgeführten Weiterverwendungen des Materials die Einwilligung des jeweiligen Rechteinhabers einzuholen.

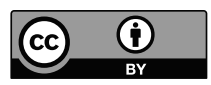

